based on routinely collected data from financial, programme budgeting as well health datasets (incidence, mortality, morbidity, and hospital activity data). We examined the relationship between the various financial initiatives like Payment by Results for secondary care, and Quality and Outcomes framework for primary care and associated disease specific outcomes within each programme category.

For each deprivation quartile, activity, cost and outcome indicator distributions will be examined using box-plots and any differences evaluated for significance. Effects of covariates on years of life lost will be assessed with a 2-step model and a generalised linear model. Using a years of life lost as a measure of health outcomes, the expenditure required to save a year of life for different age groups will be estimated.

The results from this study will be used to help improve decision making at the local level, which is particularly important in the current economic climate.

Results will be presented at the conference.

\section{5-5.2 EVIDENCE-BASED PUBLIC HEALTH POLICY: MYTH OR REALITY? A MIXED METHOD STUDY OF PUBLIC HEALTH DECISION-MAKING IN THE UK}

doi:10.1136/jech.2011.142976b.59

L Orton, * F Lloyd-Williams, D Taylor-Robinson, M Moonan, M O'Flaherty, S Capewell. University of Liverpool, Liverpool, Merseyside, UK

Introduction The potential power of public health policies emphasises the need for sound decision-making. Using research evidence to underpin public health policy has been strongly promoted; however, its implementation has not been straightforward. This study explores the use of research evidence in public health decision-making.

Methods We systematically reviewed empirical studies on the use of research evidence in public health policy (18 studies included). The identified gaps were explored through an in-depth qualitative study involving 40 interviews and three focus group discussions with senior public health decision-makers.

Results Decision-making for public health is complex. This reflects the wide determinants of health, the extensive associations between long-term conditions, and the necessity to work across sectors. A vast range of types of research evidence are used in decision-making. However, this evidence competes with many other influences. Barriers to the use of research evidence are well-described and include: decision-makers' negative perceptions of research evidence; the gulf between researchers and decision-makers; the political process of decision-making; practical time and resource constraints and limited capacity.

Ways of overcoming these barriers are less well known, and include: changing the culture of decision-making; targeting research at the needs of decision-makers; clearly highlighting key research messages; and capacity building.

Conclusion A broader conception of evidence is required to underpin public health decision-making. Achieving evidence-informed public health policy requires action by both decision-makers and researchers, in order to address the barriers identified in this study.

\section{5-5.3 PHYSICAL AND MENTAL HEALTH, SOCIAL RELATIONSHIPS, SOCIAL CAPITAL, AND HAPPINESS AMONG JAPANESE OLDER ADULTS}

doi:10.1136/jech.2011.142976b.60

\begin{abstract}
${ }^{1} \mathrm{H}$ Imai, ${ }^{*}{ }^{2} \mathrm{~N}$ Kondo, ${ }^{3} \mathrm{~K}$ Kondo. ${ }^{1}$ Yamanashi Gakuin University, Kofu, Yamanashi, Japan; ${ }^{2}$ University of Yamanashi, Chuo, Yamanashi, Japan; ${ }^{3}$ Nihon Fukushi University, Nagoya, Aichi, Japan
\end{abstract}

Introduction The purpose of this study was to examine the roles of physical and mental health on determining happiness for Japanese older adults, accounting for other individual and psychosocial factors.

Materials and Methods We used the data of Aichi Gerontological Evaluation Study, undertaken in adults aged $\geq 65$ years who were not certified as Certification of Needed Long-Term Care. The number of participants was 29546. In our 2-stage regression approach, we first estimated self-rated health using multiple variables for physical health status, including disease diagnosis, functional capacity, history of medication, body mass index, biting force, smoking, and alcohol intake, using a sex-separated ordered probit model. We then modelled happiness, measured using the PGC Morale Scale, with the estimated self-rated health and other independent variables representing mental health, socio-demographic characteristics (marital status, income, age, etc), psychosocial factors (hobby, social support, etc), and regional and individual social capital.

Results Among women, factors determining their happiness were physical health, mental health, income, age, frequency of going out, hobbies, trust of people from the local community, and general trust. Among men, factors determining their happiness were physical health, income, trust of people from the local community, and general trust. Physical health in these explanatory models was statistically significant but its effect was not large.

Conclusion Happiness may be a function of not only physical health but also mental health, as well as many individual social characteristics, including social participation and social capital. The contribution of mental health to happiness may be large.

\section{5-5.4 MATERNAL DEPRESSIVE SYMPTOMS DURING TODDLERHOOD, CHILDCARE AND CHILD BEHAVIOUR AT AGE $5 \frac{1}{2}$ YEARS}

doi:10.1136/jech.2011.142976b.61

L Giles, ${ }^{*}$ V Moore, M Whitrow, M Warin, M Davies. The University of Adelaide, Adelaide, South Australia, Australia

Introduction Disentangling the effects of maternal depression in toddlerhood from concurrent maternal depression on child behaviour is difficult from previous research. Childcare may modify any effects of maternal depression on subsequent child behaviour, but this has not been investigated widely.

Methods We examined the influence of maternal depressive symptoms during toddlerhood on children's behaviour at age $5 \frac{1}{2}$ years, and investigated if formal or informal childcare during toddlerhood modified any relationship observed.

Results Data were available from 438 mothers and their children (227 girls, 211 boys) who completed questionnaires during children's infancy, toddlerhood and at age $5 \frac{1}{2}$ years. Recurrent maternal depressive symptoms in toddlerhood was a significant risk factor for internalising, externalising and total behaviour problems when children were aged $5 \frac{1}{2} 2$ years. Formal childcare at age 2 years modified the effect of recurrent maternal depressive symptoms on total behaviour problems at child age $5 \frac{1}{2} 2$. Neither intermittent maternal depressive symptoms nor informal childcare in toddlerhood significantly affected child behaviour problems.

Conclusion Recurrent, but not intermittent, maternal depressive symptoms when children were toddlers had a longer term effect on child behaviour problems at child age $5 \frac{1}{2}$ years. As little as half a day in formal childcare at age 2 years significantly modified the effect of recurrent maternal depressive symptoms on total behaviour problems. Formal childcare for toddlers of depressed mothers is a pragmatic, supportive strategy that may have positive short and longer-term benefits for affected mothers and their children. 